\title{
Liame
}

Histoire et histoire de l'art des époques moderne et contemporaine de l'Europe méditerranéenne et de ses périphéries

25 | 2012

Les réseaux négociants dans la France méridionale (XVIII ${ }^{\mathrm{e}}$-XIX ${ }^{\mathrm{e}}$ siècles)

\section{Confiance, pouvoir et opportunisme}

Les relations de la maison Garavaque et Cusson avec ses amis du Languedoc (1759-1776)

\section{Sébastien Lupo}

\section{OpenEdition}

\section{Journals}

Édition électronique

URL : http://journals.openedition.org/liame/254

DOI : $10.4000 /$ liame. 254

ISSN : 2264-623X

Éditeur

CRISES - Centre de Recherches Interdisciplinaires en Sciences Humaines et Sociales de Montpellier

Référence électronique

Sébastien Lupo, «Confiance, pouvoir et opportunisme », Liame [En ligne], 25 | 2012, mis en ligne le 05 décembre 2012, consulté le 20 avril 2019. URL : http://journals.openedition.org/liame/254 ; DOI : 10.4000/liame.254

Ce document a été généré automatiquement le 20 avril 2019

\section{(c) (†) $\ominus$

Les contenus de Liame sont mis à disposition selon les termes de la Licence Creative Commons Attribution - Pas d'Utilisation Commerciale - Pas de Modification 4.0 International. 


\title{
Confiance, pouvoir et opportunisme
}

\author{
Les relations de la maison Garavaque et Cusson avec ses amis du \\ Languedoc (1759-1776)
}

\section{Sébastien Lupo}

1 La maison Garavaque et Cusson fondée en 1759 à Smyrne associe, tant par son capital que par ses régisseurs (à savoir Pierre-Paul Garavaque et Guillaume Cusson), le négociant marseillais Pierre-Honoré Roux à Paul Cusson, entrepreneur de la manufacture royale de Pennautier.

2 La correspondance passive de cette régie montre un commerce d'entrée consistant essentiellement en l'importation de tissus de laine languedociens, les draps s'avérant « aussi nécessaires que le pain » pour l'échelle levantine. Aussi, la construction réticulaire mise en œuvre vise-t-elle, a priori, à internaliser ce commerce dans une structure dominée par les majeurs de la maison Roux, ces derniers établissant un pont entre le marché ottoman et la production languedocienne.

Cependant, au cours des treize années d'exercice de la société, l'étude montre concrètement un réseau labile voire évanescent où les Roux changent régulièrement de fournisseurs au gré des occasions et des avantages en termes de coûts ou de réputation de chaque lieu de production du Languedoc. L'association particulière avec la famille Cusson procédant à la fois de la confiance accordée à Paul Cusson et de l'obéissance exigée de Guillaume Cusson donne lieu à un réseau organique fonctionnant difficilement et par intermittence. Les effets diffus de la crise du Levant se conjuguent aux temporalités propres à chaque société pour aboutir à la faillite de Paul Cusson en 1771 et au bouleversement structurel de la commandite smyrniote, dont la composante carcassonnaise disparaît en 1776, sans que les liens forts entre Marseille et le Languedoc aient montré leur efficacité commerciale.

$4 \quad \mathrm{Au} \mathrm{xVIII}^{\mathrm{e}}$ siècle, le commerce français des Échelles du Levant peut être considéré « comme un commerce marseillais, dans une très large mesure aux mains des négociants de la place $»^{1}$. Or, selon Charles Carrière, autour des années 1750-1760, « de nombreux rouages se grippent ", symptômes d'une " crise du Levant » assimilée à " une régression longue » du négoce transméditerranéen ${ }^{2}$. C'est pourtant entre 1756 et 1759 que la puissante 
maison Roux de Marseille décide d'installer une commandite dans l'empire du Grand Seigneur.

5 Est-ce de l'audace? De la témérité ? Le « négociant n'est pas un joueur »3. Pierre-Honoré Roux, digne représentant de cette profession, non plus. La sûreté de cette entreprise paraît même procéder d'une rationalité à même de préserver les intérêts bien comptés de la maison provençale. Il en va ainsi du choix de l'échelle. Au cours du XviII ${ }^{\mathrm{e}}$ siècle, la géographie commerciale ottomane évolue au profit de Smyrne qui supplante définitivement Constantinople dans les années $1750^{4}$. Les qualités de sa rade, sa situation en plein cœur de la côte anatolienne et son vaste hinterland drainé par les grandes routes caravanières des Proche et Moyen-Orients en font un authentique pôle de redistribution.

Dans le même temps, les Français et surtout les fabricants languedociens parviennent à imiter avec leurs londrins seconds les draps anglais et hollandais au point de les remplacer dans l'Empire ottoman dès les années $1730^{5}$. Ces tissus de laines, de qualité intermédiaire et peu coûteux, s'imposent comme le produit-phare de la consommation smyrniote. Marseille, grâce à son port franc et surtout au droit de $20 \%$ frappant les marchandises levantines qui n'y seraient pas déchargées ou dont le transport serait assuré par des navires étrangers, en assume l'exportation quasi exclusive ${ }^{6}$. Jusqu'aux années 1770 voire 1780, le volume des exportations de draps languedociens vers le Levant progresse globalement ${ }^{7}$. L'installation de commandites à Smyrne procède logiquement de l'importance de ces flux.

7 La même rationalité semble à l'œuvre en 1759 pour le choix des partenaires. La commandite smyrniote initiée par les Roux sous la raison sociale de Garavaque et Cusson associe un fabricant de draps de Carcassonne, Paul Cusson, à des négociants marseillais. Il y a ici une volonté, pour le moins manifeste, d'internaliser l'activité d'exportation de draps et d'introduire les notions de pouvoir, d'obéissance et de domination dans cette activité. Cela n'aurait rien d'étonnant dans un contexte dominé par les incertitudes du marché levantin 8 .

8 La coopération entre marseillais et languedociens dans le port anatolien a donc économiquement du sens. Toutefois, l'excellence de cette construction réticulaire n'est que théorique. Lors du renouvellement de la commandite commune après une première liquidation au bout de cinq années d'activités, Paul Cusson répond ainsi à une demande de refinancement émanant des frères Roux :

«Si le soutien de cette maizon tient a cella [des fonds supplémentaires fournis par les principaux intéressés], nous voyons qu'il faut absolument pensser a l'abandonner. Nous vous avouerons que si vous eussiez parlé comme vous nous parler nous eussions pas laisser repartir M. Gme [Guillaume] Cusson "».

9 Cette missive révèle la divergence de vues entre les intéressés au capital de la société, signe d'une réelle crise de confiance.

10 En effet, l'étude de la correspondance passive de la commandite smyrniote entre 1759 et 1776, ainsi que celles de Paul Cusson (depuis Pennautier ou Carcassonne) avec la maison Roux, toutes disponibles aux archives de la Chambre de Commerce et d'Industrie de Marseille, montre que le réseau reliant les deux commanditaires à l'échelle levantine fonctionne mal et suscite souvent l'insatisfaction des différents protagonistes. En 1769, les Cusson ne détiennent plus qu'un sixième du capital ${ }^{10}$. En 1771, le fabricant carcassonnais fait une mauvaise faillite ${ }^{11}$. Aussi, en 1772, l'intérêt de son fils Guillaume, régisseur depuis l'origine de la société, dans la nouvelle commandite de Roux n'est-il que vestigial $^{12}$; avant son éviction définitive en $1776^{13}$. 
11 Cette tentative d'intégration se solde donc par un échec relatif. La synergie entre producteur et marchand n'a pas véritablement eu lieu. D'une certaine manière, le rendezvous a été manqué. Le contexte de crise du Levant a-t-il déterminé cette issue ou bien faut-il incriminer la construction réticulaire elle-même?

12 L'examen plus précis des protagonistes puis de la réalité de leur activité commerciale dans le Levant permet de mieux comprendre la défiance qui semble progressivement contaminer leurs relations.

\section{Une commandite majeure et socialement complexe} lambda. Les Roux, s'il est encore nécessaire de les présenter, disposent de moyens financiers très importants ${ }^{14}$ et d'un réseau de correspondants qui couvre l'ensemble de l'Europe marchande, comme l'atteste la richesse de leurs archives ${ }^{15}$. En 1790, lorsqu'ils font une demande pour installer une nouvelle société à Smyrne, le secrétaire d'État évoque encore les « témoignages avantageux » qu'il a reçus à leur propos ${ }^{16}$.

Les affaires de la famille Cusson n'ont pas la même envergure. En 1758, l'entrepreneur David de la Fajeolle, auquel elle est liée, fait faillite ${ }^{17}$. Un an plus tard, Paul Cusson quitte la manufacture royale de Pennautier dont il était «le directeur technique ", pour reprendre l'expression de Claude Marquié18. Celle-ci, propriété du Marquis de Saint Pardoux, occupait alors le troisième rang dans la hiérarchie des fabriques privilégiées locales ${ }^{19}$. Ce départ n'apparaît pas comme un échec dans la correspondance de Paul Cusson. Bien au contraire, son retour à Carcassonne lui permet, selon ses dires, de produire les mêmes qualités de draps sans les contraintes de la manufacture royale, notamment en termes de personnel employée ${ }^{20}$.

relations entre les deux familles remontent aux origines de la maison Roux comme l'indique la présence dans le fonds de lettres datant de $1728^{21}$. À plusieurs reprises Paul Cusson se plaint de ne pas avoir les moyens de ses homologues marseillais, mais sans doute faut-il nuancer ces propos ${ }^{22}$. Les Roux ne sont pas des négociants à se lier à des gens qu'ils n'estiment pas être «de toute solidité ». De plus, si Paul Cusson n'a pas la même force financière, il dispose d'un capital social certain alimenté par les liens tissés avec d'autres familles de fabricants comme les Pinel, les Airolles, les Rolland ou les Astoin ${ }^{23}$.

La première trace d'un projet d'association, dépassant la simple relation commerciale entre les deux maisons, figure dans la correspondance particulière de Paul Cusson en 1756 :

«Mon fils cadet partira pour votre ville d'ici a mardi ou mecredi prochain, il aura l'honneur de vous voir très souvant et proffiter de vos bons avis et conseils. J'aurés preferé, et lui aussi, qu'il allat travailler ches vous, mais lorsque j'étois en votre ville, je vis que vous ne manquiés point du monde pour votre travail, et que votre bureau en etoit rempli, et n'aiant pu placer mon fils a la maison de M. Magy comme je l'avois projeté, et comme j'avois eû l'honneur de vous en faire part, et M. J. Bte Lassale m'aiant proccuré la maison de M. Castellane et Lacoste, et offert en même temps de prendre mon fils, et de le dresser autant qu'il se pourra au commerce de votre place, et lui donner une ouverture sur celui du Levant, j'ai crû ne devoir point le refuser (...) Je n'étois pas informé qu'on vous parlat d'un etablissement en Levant dont vous dever etre les Majeurs, si j'avois pû le prevoir j'aures surement bien prefferé qu'il eut passe a la maison dont vous deves etre les majeurs si cella s'etoit pu, mais de la façon que nous avons faites les conditions avec M. Castellane et 
Lacoste, mon fils ne sera point abstreint en rien, et lorsqu'il sera dans ce païs la, ou

avant même d'i aller, il sera libre de changer de maison s'il le veut $»^{24}$. mais il promet de ne rien négliger pour procurer aux régisseurs smyniotes "autant d'amis et de parents qu'il se pourra pour leur adresser des draps » et de produire à Carcassonne des tissus qui « seront propres pour cette échelle $»^{30}$. De plus, il fait profiter la société de ses connaissances concernant les producteurs languedociens, les marseillais n'hésitant pas lui demander des informations sur ses voisins ${ }^{31}$. Le principe de parité structure la commandite. Paul Cusson et Pierre-Honoré Roux financent chacun pour moitié le cautionnement de la régie ${ }^{32}$ montant à $60.000 £$ t, un des plus importants du Levant $^{33}$. De même, le capital de 12.000 piastres est partagé équitablement entre PierreHonoré Roux, Guillaume Cusson, même si dans les faits son père paie son contingent, et Pierre-Paul Garavaque ${ }^{34}$. Ce dernier, un jeune négociant marseillais de vingt-cinq ans, assume la charge de régisseur principal ${ }^{35}$. Il a toute la confiance des majeurs marseillais puisqu'il a longtemps travaillé dans leur comptoir - Paul Cusson parle même d'un « temps infini » - et qu'il est l'ami du fils aîné de Pierre-Honoré Roux ${ }^{36}$. Guillaume Cusson doit, quant à lui, tenir la caisse et faire profiter la société de sa connaissance des langues acquise au cours de ses trois années passées à Constantinople ${ }^{37}$. L'équipe de régisseurs symbolise bien l'hybridation entre les deux familles négociantes. La maison Roux, véritable charnière du réseau, envoie à Carcassonne la copie de ses courriers destinés à Smyrne $^{38}$. Et elle fait suivre les lettres qui en proviennent, après en avoir pris connaissance $^{39}$. Tout concourt à la transparence et à l'établissement d'un négoce mutuellement fructueux.

\section{Le commerce languedocien de Garavaque et Cusson}

Le commerce d'entrée domine l'activité de la commandite. Les draps, jugés aussi «nécessaires que le pain », représentent les trois quarts des marchandises reçues selon les comptes de la sociététe. Cependant, l'étude plus précise des flux de ballots ne peut reposer sur ces seules pièces comptables. D'une part, la série comporte de nombreuses lacunes notamment en 1760,1762, 1768 et suivantes. D'autre part, ces pièces ne 
concernent que la maison Roux et tous les envois faits par les autres amis de la régie n'y apparaissent pas. Le recours à la correspondance passive s'impose, avec son lot de difficultés propres. Les régisseurs ne respectent pas de plan précis dans leurs lettres. Les informations relatives aux draps peuvent se trouver partout dans les courriers. Leur qualité s'avère également très variable. Dans le meilleur des cas, le nombre de ballots, la nature du tissu, le nom du fabricant, le lieu de production, les numéros de ballots, les nom $\mathrm{du}$ capitaine ainsi que de son bateau responsables du transport et les personnes intéressées avec le montant de leur participation peuvent figurer. Les occurrences de ces descriptifs complets sont rarissimes et les négociants font souvent preuve de laconisme en n'évoquant que le nombre de ballots et le fabricant. Les évidences des régisseurs gênent l'historien. Au surplus, une même pièce de drap peut apparaître jusqu'à quatre reprises dans les lettres: tout d'abord lorsque les smyrniotes sont avertis de son expédition, quand les draps sont effectivement reçus dans l'échelle, puis vendus et enfin, quand Garavaque et Cusson expédie le compte de vente finalisant l'opération. Le croisement de ces informations avec les pièces comptables permet, dans une certaine mesure, d'évaluer la pertinence de la série. Aussi, suivre le mouvement des draps ne constitue-t-il pas une sinécure.

21 Un tel décompte met au jour le réseau des fournisseurs. Deux éléments le caractérisent : leur grand nombre et la diversité de leur localisation. Dans les magasins de Garavaque et Cusson se côtoient les productions de Miailhe, Andrieux, Benazet, Brouzet, Montlaur, Aussenac, Astoin, Pattot, Pailhoux, Jullien, Cavailhès, Pinel, Lannolier, Albouy, Anduse, Bosc, Baron, Arnaud, Gazelle, (liste non exhaustive) provenant majoritairement de Carcassonne mais aussi de Saint-Chinian, Limoux, Saint-Pons, Clermont, Saissac, Chalabre, Mas-Cabardès, Lodève... Au cours des douze années d'existence de la régie aucun partenaire ne se détache. Les majeurs ou leurs amis cherchent le meilleur prix d'achat avant la qualité des draps ou des assortiments. Le recours fréquent à des établissements situés en dehors de Carcassonne obéit à cette logique. Moins prestigieux, ces draps présentent un prix d'achat en France moindre et peuvent s'approcher des cours des londrins de Carcassonne dans le Levant. C'est notamment le cas des tissus de SaintChinian ${ }^{41}$. Aussi, l'intégration marseillo-carcassonnaise promise ne paraît pas évidente.

Certes, on retrouve avec une certaine régularité, parmi ces fabricants, les proches de la famille Cusson comme Astoin, Ayrolles ou Sabatier, mais pour des volumes faibles. De plus, ces deux derniers se concentrent sur les londres larges ou ordinaires, des qualités qui connaissent un discrédit croissant à Smyrne. Ils ne constituent donc pas des partenaires déterminants de la régie. La mise en série des informations relatives aux draps pour la période 1759-1772 révèle surtout, pour les cinq premières années, un étiage particulièrement bas des envois opérés par Paul Cusson. En dehors de 1759, année d'installation, ces derniers ne représentent même pas $10 \%$ du volume de tissus traité par la commandite.

Cela s'avère d'autant plus notable que le travail à la commission régresse significativement au cours de la même période (voir documents en annexe). Pour obtenir des marchandises, les majeurs, ainsi que leurs régisseurs, doivent endosser une partie des risques. À partir de 1762, un nombre sans cesse croissant d'envois se font en compte à tiers associant le producteur ou un partenaire languedocien, les Roux et la maison smyrniote. Cette particularité provoque de fortes tensions dès la fin de la guerre de Sept Ans, lorsque la place levantine est saturée de marchandises vendues à vils prix. « Notre maison est montée différemment de toutes celles du Levant qui ne travaillent pour 
l'ordinaire qu'en comm ${ }^{\text {on }}$ [commission] et alors les intéressés ne sont obligés a rien, mais la notre travaillant plus pour son compte, chaque intéressé devrait faire un travail egal ", écrit Pierre-Paul Garavaque en $1763^{42}$.

Le conditionnel vaut accusation et l'examen des chiffres se révèle accablant. Le décalage entre les majeurs et le fabricant de draps est manifeste, d'autant plus que les Roux s'activent dans toutes sortes d'opérations (expéditions de bateaux, arbitrage sur les monnaies, envois de marchandises des îles) ce qui n'est que très marginalement et très tardivement le cas pour Paul Cusson. Les Roux assurent l'essentiel du commerce d'entrée de la commandite. Après la première liquidation, la donne évolue, l'investissement cussonien dépasse le seuil de $10 \%$ selon trois modalités différentes. En 1765, il s'agit d'un effort propre. En 1766, les deux tiers des ballots concernant Paul Cusson relèvent d'un compte à demi avec sa belle-famille Astoin. Suite à ces efforts, plus conformes aux engagements de 1759 , son investissement prend la forme d'une prise de participation d'un tiers dans les expéditions pilotées par les Roux. À l'exception de l'année 1769, marquée par un envoi de 32 ballots produits par un Cusson et dont le recouvrement est géré par Paul, on ne peut faire aucun lien entre ces traités de draps et le réseau familial de ce dernier. Les espérances de 1759 ont été déçues et les rouages de l'échange rapidement grippés.

L'ingratitude du commerce constitue sans doute le lieu commun le plus éculé des correspondances négociantes. Cependant, les difficultés s'amoncellent précocement pour les régisseurs. À l'entrée, les draps peuvent présenter un manque d'aunage, des « piqûres de vers ", des parties plus étroites, trop claires ou plus simplement un assortiment de couleurs qui rebute les acheteurs. Ces défauts s'avèrent particulièrement fréquents au début de la commandite. Les " amis » tendent à considérer Garavaque et Cusson comme un moyen opportun pour se débarrasser de tous les vieux draps invendus qu'ils ont en réserve. Les régisseurs s'en plaignent d'ailleurs :

« Nous avons essuyé toute sorte de désagrément au sujet des picures de vers des draps que nous avons vendu et que nous avons en magazin, les acheteurs en sont telement frapés qu'ils visitent pièce par pièce tous les draps que nous leur vendons, cella est capable de discréditer notre magazin ${ }^{43}$.

Aussi, pour s'assurer de la qualité des draps et faire teindre à leurs assortiments, régisseurs et majeurs marseillais doivent faire produire, ce qui signifie prendre un intérêt aux draps et participer aux risques.

Le retour des fonds pose également problème. Les languedociens, sans faire exception, réclament des retours prompts en lettres de change. Or, les cours "sur la chrétienté " sont souvent très dégradés et peuvent générer des pertes approchant $20 \%$, ce qui absorbe le bénéfice d'entrée, voire plus ${ }^{44}$. En outre, le marché smyrniote souffre d'un manque de liquidités. La recherche de papier "de toute solidité» peut s'éterniser ${ }^{45}$. Enfin, les circonstances défavorables peuvent conduire les régisseurs à faire des retours en marchandises. Pis-aller pour éviter une nouvelle baisse des cours ou échapper à la dégradation des draps en magasin, ils aggravent le risque couru par les partenaires et retardent la réception effective de leurs fonds. De plus, les métropolitains se montrent rarement compréhensifs face aux dilemmes vécus par les smyrniotes. À ce titre, on peut lire, sous la plume de Paul Cusson le $1^{\mathrm{er}}$ août 1764, relativement à Garavaque et Cusson, que « ces messieurs aiment fort les retours en marchs [marchandises] parce que cela leur porte $4 \%$ au lieu de deux quand ils font des remises ", c'est-à-dire des retours par lettres de change ${ }^{46}$. Il s'agit d'une accusation lourde d'autant que, parmi «ces messieurs», se 
trouve Guillaume Cusson, son fils décrit auparavant comme « sage et travailleur », à l'égal de Pierre-Paul Garavaque ${ }^{47}$.

Les écueils du commerce levantin ont mis à mal la qualité des relations interpersonnelles entre les majeurs et leurs régisseurs. Le maître d'œuvre de premier rang pour le commerce de la commandite se situe à Marseille et, dans une large mesure, les Cusson ont laissé les Roux la faire fructifier. Garavaque et Cusson révèle ainsi la complexité des rapports sociaux et les divergences d'intentions de ses différents protagonistes.

\section{Une régie de défiance}

La crise, due à la saturation du marché smyrniote au lendemain de la guerre de Sept Ans, pose avec acuité le problème de l'implication des partenaires. Lors des discussions relatives au renouvellement de la commandite, Pierre-Paul Garavaque sonne la charge : «Pendant les 5 dernières années, nous pouvons dire avoir été les dupes de M. Paul Cusson $»^{48}$. Le jeune marseillais est non seulement régisseur, mais aussi intéressé au capital de la commandite, au même titre que les Roux ou la famille Cusson. En définitive, cette dernière doit retirer les mêmes bénéfices que ses partenaires lors de la liquidation, alors que le volume d'affaires généré est incomparable à celui des marseillais (voir les documents en annexe). Pour Garavaque, il s'agit d'une authentique stratégie de passager clandestin.

En effet, la structure de la commandite, dans laquelle les majeurs ont la même part de capital et un régisseur de leur choix chacun, laisse croire à une relation qui n'a rien de hiérarchique. Les maisons de Carcassonne et de Marseille échangent depuis longtemps et elles ont choisi de s'associer librement. On peut donc considérer cette relation comme horizontale. Dans cette optique, on comprend que dans les esprits marseillais, les Cusson manquent aux obligations de coopération, de solidarité et de confiance qui animent ce type de rapports sociaux ${ }^{49}$.

Or, l'étude de la correspondance de Paul Cusson montre qu'il est persuadé de sa bonne foi et de son honnêteté. Dès l'installation de la commandite à Smyrne, son attitude prouve qu'il considère son rapport à Pierre-Honoré Roux dans une logique plus verticale, plus hiérarchique. Outre la différence de moyens, le drapier avoue ne pas connaitre le " commerce de cette échelle $»^{50}$. La lecture de la correspondance révèle l'exactitude de cette assertion. Les régisseurs estiment que les draps de Paul Cusson sont trop chers et trop beaux pour le marché smyrniote ${ }^{51}$. De plus, le drapier carcassonnais ne semble pas privilégier la commandite. En 1761, la lettre du 4 mai fait état de plusieurs défectuosités majeures dans les londres larges de Sabatier qu'il a expédiées ${ }^{52}$. Plus surprenant, on peut lire dans le pli du 24 juin 1762 :

«M. Paul Cusson \& Fils nous ont marqué (...) qu'ils avaient vendu tous les L.S.

[londrins seconds] de leur fabrique, ainsi que les L.L. [londres larges] de Sabatier,

attendu qu'ils trouvaient plus d'avantage a les vendre a Marseille qu'a nous les

expédier. Cela étant, nous aurons peu d'envois a attendre de leur part cette année

(...) puisqu'ils ne comptent de travailler que dans les bons temps $»^{53}$.

Comme le confirment les documents annexes, Paul Cusson a souvent fait de pareils calculs les premières années. Il ne participe pas non plus aux expéditions à Constantinople de draps de la manufacture de Pennautier dirigée par son ancien commis, Tarbouriech. Pourtant, les retours devant s'effectuer via Smyrne, il y avait là l'occasion de donner du travail aux régisseurs. Ce retrait insupporte d'autant plus ses partenaires que 
l'existence même de Garavaque et Cusson induit des frais importants, notamment de résidence et de location de magasins. Paul Cusson ne paraît pas se sentir concerné, ni même redevable. Dès le 3 décembre $1763^{54}$, Guillaume Cusson étant en voyage, Garavaque estime dans une lettre de société que ce serait « de bonne justice » que tous les intéressés fassent également travailler sa société.

Pour ce faire, il échafaude plusieurs projets afin d'impliquer davantage les carcassonnais. Sa première proposition en date du 31 mars 1764 consiste à fixer un seuil minimum d'affaires d'entrée, de l'ordre de 3600 piastres pour payer les charges de la société. En cas de manquement, la différence serait débitée dans le compte courant de l'intéressé. Le 28 avril 1764, il affine son projet :

" Je crois avoir etabli les conditions les plus raisonnables et les plus equitables entre nous, en demandant une obligation de la part de chaque intéressé de $20 \mathrm{~m} / \mathrm{Pa}$ [mille piastres] l'année pour pouvoir nous assurer au moins les dépenses de notre maison et dans le cas que quelqu'un de nous ne remplit pas son contingent il seroit debite en cte court [compte courant] à la fin de l'année de la commission sur la somme qui manquerait de ces $20 \mathrm{~m} / \mathrm{Pa} \|^{55}$.

Le 26 mai 1764, Paul Cusson refuse les conditions proposées par Garavaque en les jugeant "très absurdes" et en affirmant nettement: "nous ne sommes rien ${ }^{56}$. Avec un tel présupposé, la situation peut paraître normale. Les Roux font l'essentiel du commerce de Garavaque et Cusson, car ils sont les majeurs. Il y a donc une hiérarchie claire dans les esprits carcassonnais, mais cette relation verticale ne se double d'aucun principe de domination ou d'obéissance ${ }^{57}$. Le 21 juillet 1764, Paul Cusson accepte d'entrer dans un compte à tiers mais refuse de faire passer des draps. C'est alors qu'il brandit la menace de retirer Guillaume Cusson du Levant, préférant ce retrait à la perte d'argent assurée que causeraient pareilles affaires ${ }^{58}$.

Pierre-Paul Garavaque livre son baroud d'honneur le 16 octobre 1764. Il propose comme la "base de [son] établissement", l'expédition de tous les draps de Paul Cusson à Constantinople, les retours passant par ses mains ${ }^{59}$. "Nous ne croions pas que Mess. [Messieurs] Paul Cusson et Fils refusent de retenir $1 / 3$ d'intérêt à leurs draps et alors les 2/3 seroient partagés entre l'ami de Cple [Constantinople], vous [Roux] et nous [Garavaque et Cusson] $\aleph^{60}$. Hypothèse infondée, dès le 22 septembre, le drapier avait refusé de prendre la moindre participation à ses draps s'ils étaient expédiés au Levant, et ce, quelle que soit leur quantité, jugeant l'époque défavorable ${ }^{61}$. Et quand bien même, ses projets consistent à vendre à Constantinople ou au Caire pour donner une réputation à ses productions, les retours se faisant en lettres de change. Smyrne demeure dans l'angle mort de son activitéé .

Le concept sociologique d'opportunisme, « cette combinaison de l'intérêt personnel et de la tromperie ", même si le comportement de Paul Cusson n'est pas foncièrement malin, semble adapté à la situation ${ }^{63}$. Il ne choisit pas clairement sa position dans la structure réticulaire de Garavaque et Cusson. Du point de vue du capital, il est l'égal des Roux. Cependant, il admet aussi leur domination car ils sont juridiquement les majeurs et, à ce titre, il les laisse s'escrimer à faire fructifier la commandite. Mais il refuse de leur obéir ou même de se montrer solidaire en excipant ses intérêts personnels, niant tout rapport qu'il soit vertical ou horizontal. En cela, il y a une forme de tromperie. On ne peut participer aux bénéfices sans être partie prenante, d'une manière ou d'une autre. Guillaume Cusson, qui n'a jamais été en mesure de rembourser son père des 4.000 piastres de mise de fonds, 
n'est qu'un prête-nom. Il n'échappe pas pour autant à la défiance de ses partenaires marseillais ${ }^{64}$.

Entre les deux régisseurs, le travail en bonne intelligence n'advient qu'au bout de huit années. Lors du voyage en Languedoc de Guillaume en 1763, le jeune associé marseillais trouve dans ses lettres un exutoire. La première occurrence de reproches se montre relativement modérée. "Cusson fait bien tout ce qu'il peut mais on ne peut pas s'en rapporter entièrement sur lui », note-t-il après avoir évoqué les compétences du nouveau commis recruté en $1763^{65}$. La charge se précise quelques mois plus tard:

«Tout ce que vous me dites de M. Cusson n'est point nouveau pour moy, ni pour tous ceux qui le connaissaient bien dans ce paÿs et par conséquent j'étais faché qu'il allat se faire connoitre à Marseille où il n'aura pas donné une grande idée des regisseurs de votre maison. Je vous dois au reste des remerciements de ce que vous avés bien voulû en suggerer tout autre de moy a vos amis ${ }^{66}$.

Il est vrai que la lecture des échanges épistolaires entre la maison marseillaise et Guillaume Cusson, lors de son voyage en France, laisse apparaître quelques expressions qui détonnent dans une correspondance négociante généralement policée. Ainsi, dans sa lettre du 20 juillet 1763, depuis le lazaret, il écrit en apostille: "Vous me fairés bien plaisir de repondre a ma lettre $\aleph^{67}$. Cette drôle d'impression trouve une confirmation dans celle du 29 juillet. À propos d'un assortiment envoyé de Smyrne qu'il doit transmettre à sa famille, il écrit : « si cella ne vous derrengeoit pas beaucoup de vos occupations vous me feriés bien du plaisir de me l'envoier par le retour du porteur du presant $»^{68}$. Il ne s'agit pas de manquement à la bienséance, mais on sent poindre de l'impatience qui parait adéquate à la remarque précédente de Garavaque. Aux plaintes de Roux concernant le peu d'affaires provenant de ses amis languedociens, il répond, en des termes bien plus tranchés que son père, que les marseillais seraient «bien les maitres » de mettre fin à la commandite ${ }^{69}$.

En fait, les reproches convergent depuis Marseille et Smyrne. Comme on l'apprend dans la lettre du 9 février 1764, Guillaume aurait un peu trop claironné que, par sa venue, il procurerait de nouveaux fournisseurs à sa société, là où les Roux avaient échoué ${ }^{70}$. Son peu de modestie s'associe à son caractère dépensier. Pour Garavaque, Cusson ne lui sert "presque à rien et fait augmenter de beaucoup les dépenses » de la maison ${ }^{71}$. Aussi oppose-t-il une fin de non-recevoir lorsqu'il apprend que le second fils cadet de Paul Cusson doit être envoyé à Smyrne, "n'ayant nullement besoin de nouveaux mangeurs et de membre inutile ${ }^{72}$. La défiance de Garavaque provient également de la compétence de son associé, semble-t-il à raison. Ainsi, suite à des erreurs répétées dans la tenue de la caisse, Garavaque décide de s'en charger et " de l'ôter à Cusson ", jugeant que pour éviter d'être dupé il faut une bonne connaissance de la place ${ }^{73}$. Or, "il n'est pas trop facile d'aprendre à lire et à écrire les langues de l'Orient, c'est là l'ouvrage de 10 ans des jeunes de langue à Constantinople qui ne font autre chose ». S'agit-il d'une remarque ironique à propos de Cusson qui a, en effet, séjourné à Constantinople avant de rejoindre la place de Smyrne, mais qui s'y fait aussi facilement berner ? Écarter Cusson doit permettre, en tous les cas, de mettre fin aux nombreuses "erreurs dans [leur] comptoir». Lorsque le régisseur principal échafaude le projet d'envoyer les draps de Paul Cusson à Constantinople, il envisage d'expédier également son fils à la capitale pour représenter les intérêts de la société. Espoir rapidement déçu, Paul Cusson s'oppose à ce départ. Il ne reste à Garavaque qu'à prendre patience face à « la bonne volonté » de son partenaire qui "se réduit à peu de chose attendu qu'il manque par les principes ». On est assez loin de 
«la bonne union et de la bonne concorde » entre régisseurs évoquées dans le Mémoire instructif rédigé par les majeurs en $1759^{74}$.

Selon nos sources, Guillaume n'est pas seulement victime de critiques. Sa lettre particulière du 22 août 1764 montre que l'antipathie était réciproque :

«Il est bien facheux pour moi qu'un malentendû m'occasionne de si vifs reproches de la part de mon père, et il faut que vous lui aiés fortement écrit contre moi, car il me donne un furieux savon par sa lettre du 6 juillet (...) Je vous avoue que je ne me serais point attendu a cella de votre part $»^{75}$.

41 En l'occurrence, le cadet des Cusson a conclu un accord avec Ayrolles pour faire passer des draps à Constantinople avec une participation de la commandite, sans en avertir son associé ni ses majeurs ${ }^{76}$. De même, pour faire cesser la résistance des régisseurs contre la baisse drastique de leurs frais, les Roux demandent au père de raisonner son fils. Le 3 octobre 1764, le patriarche annonce avoir conduit à résipiscence son enfant trop prodigue ${ }^{77}$. Un tel recours à des pressions familiales pour faire plier les régisseurs n'est cependant pas spécifique. Dans ce réseau négociant, les liens familiaux ou amicaux ne déterminent pas la confiance. Pierre-Paul Garavaque subit le même traitement et demande dans une lettre particulière que les Roux cessent d'impliquer sa mère, qui n'a rien à voir avec le commerce levantin ${ }^{78}$.

Le cas de Guillaume Cusson illustre donc, à nouveau, la complexité des rapports sociaux à l'œuvre dans la commandite. Peu compétent, dépensier, peu amène, voire fat, ce régisseur aurait pu être renvoyép ${ }^{79}$. Toutefois, sa part, même fictionelle, au capital de la société interfère dans la relation verticale avec ses majeurs et explique peut-être aussi son ton parfois altier. On comprend dès lors que les Roux en soient réduits à faire usage d'une autre relation verticale, celle qui relie un père à son fils incapable de rembourser sa mise de fonds. Régisseur dispensable mais (fils d') associé intouchable.

Ce n'est qu'en fin de période que la situation semble se normaliser. Ainsi, avant de quitter

le Levant, Pierre-Paul Garavaque parle de Cusson comme d'« un ami », certes aux capacités négociantes limitées, auquel il est attaché et pour lequel il n'a « aucune raison de vouloir [s'en] séparer $»^{80}$. De même, la correspondance privée, à partir de 1768, entre le fils de Paul Cusson et les Roux n'est que démonstrations d'obéissance et d'excuses pour ne pas avoir suivi leurs conseils dans ses tendres années.

«Si ma conduite passée a pu vous deplaire, je vous suplie de vouloir bien l'oublier et soiés assurés messieurs que désormais ma conduite sera analogue à votre sage façon de penser et pleut a Dieu l'eussai-je adobtée dans le passé j'en serrois plus content et plus tranquille $»^{81}$.

Guillaume en régisseur docile, Paul Cusson, même si ce n'était pas son engagement initial, participant dans un compte à tiers aux expéditions de draps : on pourrait croire qu'au cours de la deuxième société Garavaque et Cusson, les relations s'apaisent. D'ailleurs, lors de la refondation de la régie en 1772, sous le nom de "Cailhol, Cusson et Compagnie ", Guillaume Cusson est charitablement conservé malgré son impécuniosité ${ }^{22}$.

Cependant, un faisceau d'indices atteste le contraire. Lors de la faillite de Paul Cusson en 1771, on trouve la trace d'un litige concernant un billet du Canada. Le languedocien se défend en écrivant : « vous nous permettrés de vous répéter ce que nous eûmes l'honneur de vous dire lorsque vous nous menaçates de nous attaquer en parlement ${ }^{83}$. Or, les négociants fuient en général la justice royale, coûteuse et longue, pour lui préférer des arbitrages entre pairs. Cette menace dénote un niveau de tensions assez rarement vu dans les correspondances entretenues par les Roux. De même, si le cadet des Cusson est 
conservé en 1772, les majeurs marseillais le privent de la signature, une première en treize années ${ }^{84}$. Il faut une intervention de l'autre régisseur, Cailhol, pour que cette décision soit révisée ${ }^{85}$. C'est par le même canal qu'il sera définitivement disgracié en 1776. Privé du soutien familial et ayant épuisé la patience de ses majeurs, Guillaume Cusson est congédié sans autre forme de procès. Cailhol s'avoue même surpris « de la promptitude de la résolution » prise à Marseille ${ }^{86}$. L'aîné des fils Cusson, sollicité pour une médiation, ne peut qu'écrire après les explications de Roux : «il y a des caractères qui ne changent jamais (...) l'on est malheureux quand dans des familles l'on a des pareilles sujets ${ }^{87}$. Ainsi, dans sa propre famille, les qualités de Guillaume ne font pas mystère. D’ailleurs, cette éviction inquiète la régie, non pas à cause des compétences du carcassonnais mais pour sa réputation, car «les divorces entre associés font toujours un mal infini dans l'esprit des correspondants et ne peuvent devenir que tres nuisible meme aux maisons les mieux etablies $»^{88}$. Par conséquent, bien plus que la force des liens à l'intérieur de la triade Roux-régisseur principal-Cusson, « la configuration générale des réseaux sociaux », dans lesquels ces acteurs étaient situés, semble expliquer la persistance de cette construction réticulaire après $1769^{89}$.

La crise du Levant n'a pas fini de dévoiler ses arcanes. Il est certain que la dégradation des changes, en ralentissant ou en dénaturant les retours, les conflits, en perturbant les échanges et en favorisant les déséquilibres, notamment la saturation des marchés levantins, ont contribué à refroidir les ardeurs languedociennes. Cependant, l'étude a montré que Paul Cusson n'a jamais essayé d'adapter sa production aux demandes smyrniotes. Au mieux, a-t-il consenti à prendre une participation dans des traités établis par les Roux avec d'autres producteurs languedociens entre 1767 et 1769. À l'échelle de notre réseau, il y a donc également un problème de qualité de l'offre. En refusant de réellement coopérer à l'intérieur de la triade qu'il forme avec les Marseillais et leur commandite levantine, Paul Cusson s'enferme dans les stratégies mises en œuvre avant l'établissement de Garavaque et Cusson: privilégier Constantinople, alors que sa consommation régresse, vendre à Marseille, plutôt que faire travailler la société dans laquelle il a investi. L'échec relatif de l'association marseillo-carcassonnaise semble bien procéder d'un phénomène de "dépendance de sentier », tel que le définit Douglass North, c'est-à-dire comme « un ensemble de contraintes imposées aux possibilités de choix du présent à cause des expériences historiques du passé $»^{90}$.

Or, la structure institutionnelle de la commandite a contribué à ce processus. En entretenant la fiction que Guillaume Cusson participait au capital de la société, elle privait les majeurs marseillais d'une part de leur autorité et les forçait à composer avec un régisseur inefficace, alors que l'objectif consistait à impliquer un fabricant de draps. Par la même, ce dernier, à chaque retournement de conjonctures, pouvait justifier son inertie. L'alpha et l'oméga de la solidarité familiale résidant dans la préservation des intérêts de la maison de Carcassonne, Paul Cusson a tôt fait de considérer qu'il n'était rien pour la commandite smyrniote. L'anomalie remonte, par conséquent, à la création de la société : en le sollicitant en 1759 , les Roux n'avaient sans doute plus à l'esprit le courrier de 1756 où l'opportunisme de leur interlocuteur pointait déjà (ou alors n'en avaient-ils pas intégré tous les tenants et aboutissants). À la différence de moyens et de statut juridique, s'ajoute l'ambigüité, scellée dans les conventions de 1759, du positionnement des Cusson dans la structure de la commandite. Une fois installée, il devenait socialement plus délicat de briser définitivement cette collaboration. L'empressement de Cailhol à justifier le renvoi de Guillaume auprès de ses amis négociants de la nation française de 
Smyrne montre à quel point le problème de la réputation entrait en ligne de compte ${ }^{91}$. Il explique sans doute la permanence de la triade Paul Cusson-Roux-Garavaque et Cusson, en dépit de la défiance qui anime leurs relations. Les négociants savaient bien qu'on ne sort de l'ambiguïté qu'à ses dépens.

\section{ANNEXES}

Graph. 1 : évolution des importations de draps languedociens impliquant la maison Garavaque et Cusson de Smyrne (1759-1772)

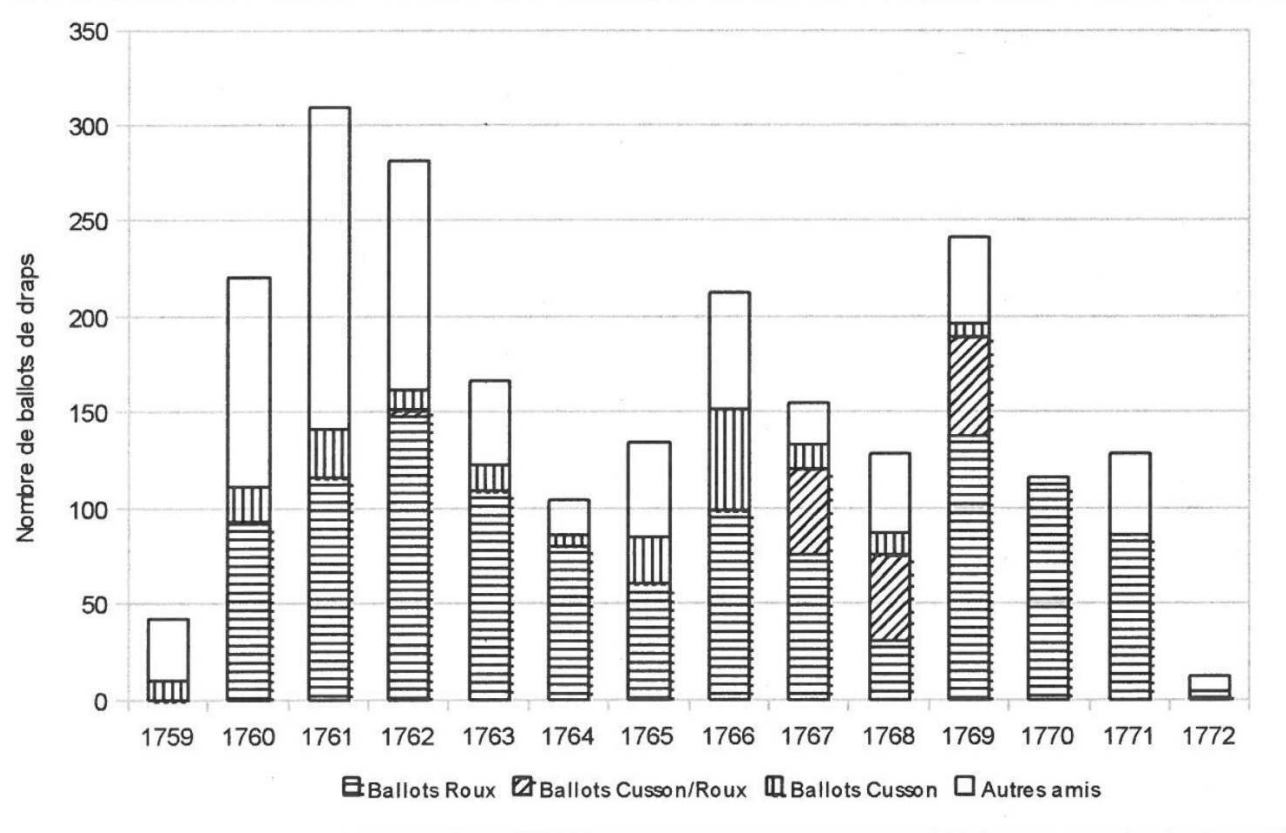




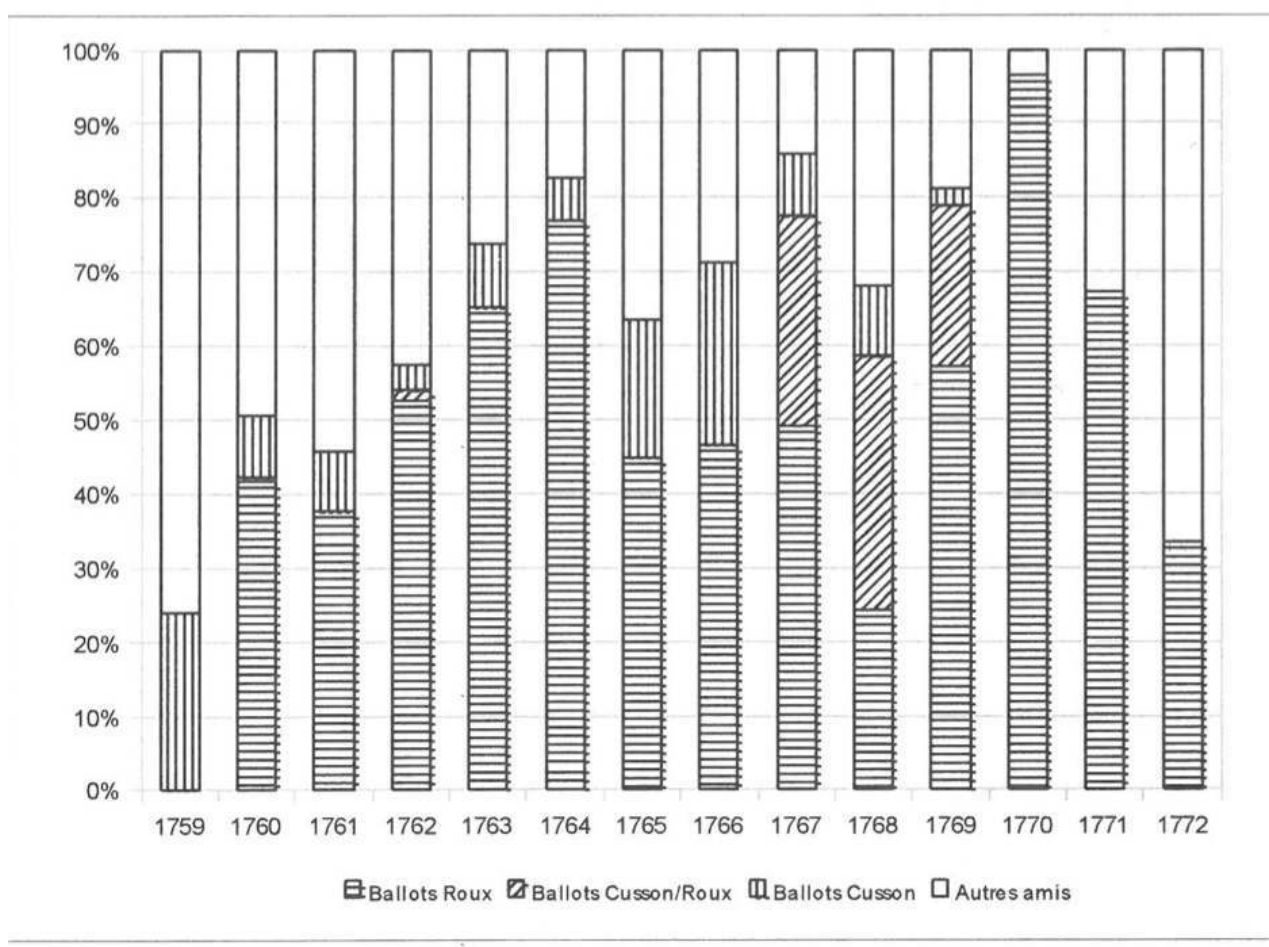

\section{NOTES}

1. Charles CARRIÈRE, Négociants marseillais au XVIII ${ }^{\mathrm{e}}$ siècle - Contribution à l'étude des économies maritimes, Marseille, Institut historique de Provence, 1973, p. 320.

2. Charles CARRIÈre et Marcel COURDURIÉ, «Un sophisme économique. Marseille s'enrichit en achetant plus qu'elle ne vend (Réflexions sur les mécanismes commerciaux levantins au XviII siècle), Histoire, Économie et Société, 1984, p. 9.

3. Charles CARrière, Marcel Courdurié, Michel GutSATZ et René SQUARZoni, Banque et capitalisme commercial - La lettre de change au XVIII ${ }^{\mathrm{e}}$ siècle, Marseille, Institut historique de Provence, 1976, p. 152.

4. Edhem ELDEM, Le commerce français d'Istanbul au dix-huitième siècle, thèse de doctorat préparée sous la direction de Robert Mantran, Aix-en-Provence, 1989, p. 46.

5. Katsumi FuKASAWA, Toilerie et commerce du Levant d'Alep à Marseille, Paris, Éditions du CNRS, 1987, p.125.

6. Charles CARRIÈre, Négociants... op. cit., p. 319.

7. Charles CARRIÈRE et Michel MoRINEAU, « Draps du Languedoc et commerce du Levant au XVIII siècle ", Revue d'Histoire économique et sociale, 1968, p. 117.

8. Mark GranovetTer, Sociologie économique, Paris, Seuil, 2008, p. 236.

9. Archives de la Chambre de Commerce et d'Industrie de Marseille-Provence [désormais ACCIM], LIX/205, lettre du 21 juillet 1764.

10. ACCIM, LIX/1038, convention du 7 avril 1770. 
11. « Nous voyons que Mess. Paul Cusson étaient a la veille de terminer leurs affaires et qu'ils ne pouvaient donner que $25 \%$ à leurs créanciers ». (ACCIM, LIX/744, lettre particulière de Guillaume Cusson du 10 avril 1771).

12. Cailhol écrit à ce sujet : «Considérés je vous prie qu'il est assés malheureux dans celle-ci [la société Cailhol, Cusson et Compagnie] de voir son interet reduit de la moitié ». (ACCIM, LIX/730, lettre particulière de Cailhol du 9 janvier 1773). Cela signifierait donc que Guillaume Cusson ne détient plus qu'un douzième du capital de la commandite.

13. ACCIM, LIX/731, lettre du 6 juillet 1776.

14. Pour ne citer qu'un chiffre, l'avoir liquide de Pierre-Honoré Roux est évalué à $500.000 \mathrm{ft}$ en 1762. Voir Ferréol ReBufFat, Répertoire numérique des archives de la Chambre de Commerce et d'Industrie de Marseille, Marseille, 1965, p. 89.

15. La série LIX aux ACCIM comporte près de neuf cents liasses de lettres. Cf. Gilbert BuTI, "Stratégies marchandes au temps des troubles et des incertitudes. Le cas de la France méditerranéenne au XVIII ${ }^{\mathrm{e}}$ siècle ", The Historical Review-La Revue Historique, $\mathrm{n}^{\circ} 7$, Institut de recherches néohelléniques (FNRS), Athènes, 2010, p. 30.

16. ACCIM, J/64, lettre du 15 février 1790.

17. Claude MARQUIÉ, L'industrie textile carcassonnaise au XVIII ${ }^{\mathrm{e}}$ siècle : étude d'un groupe social, les marchands-fabricants, Carcassonne, Société d'études scientifiques de l'Aude, 1993, p. 201.

18. Ibid., p.104.

19. Ibid., p.95.

20. «C'est de quoi nous serions bien fachés quand a nottre fabrique, nous pourrions dire avec vérité qu'elle nous a donné de la perte et cella depuis deux ans et sy nous n'avions pas ete embarrasses d'une manuf[acture] ou il faloit y faire travailler les ouvriers, nous aurions sesse de fabriquer et eviter bien de pertes ». (ACCIM, LIX/204, lettre du 30 juin 1759).

21. Voir ACCIM, LIX/210, lettres de David et Cusson.

22. ACCIM, LIX/525, lettre particulière de Paul Cusson du 24 janvier 1759.

23. Claude MARQUIÉ, op. cit., p. 274.

24. ACCIM, LIX/203, lettre particulière du 4 décembre 1756.

25. Curieusement, la plus ancienne pièce contenue dans la liasse LIX/1038 des archives de la Chambre de commerce de Marseille fait état d'un Michel Cusson qui n'est attesté nulle part dans la correspondance.

26. ACCIM, LIX/203, lettre particulière du 4 décembre 1756.

27. ACCIM, LIX/525, lettre du 24 janvier 1759.

28. Ibid.

29. "J'ai reçu la lettre que vous m'avés fait l'honneur de m'écrire le 2 du courant, dans laquelle vous avés la bonté d'entrer dans un détail très circonstancié et qui me fait voir avec raison que l'établissement pour M. Garavaque et pour mon fils est préférable de toutes les façons a Smyrne plustot qu'a Constple [Constantinople] ou il n'y aurait d'autre raison pour la preferer que celle de la garantie de la caisse nationale qui est bien balancé et au dela par la lenteur des ventes des draps ». (ACCIM, LIX/525, lettre du 7 février 1759).

30. Ibid.

31. C'est le cas par exemple de Brouzet dans la lettre du 10 janvier 1759, décrit comme « un homme très exact et loyal ». (ACCIM, LIX/525).

32. ACCIM, LIX/525, lettre du 24 janvier 1759.

33. Charles CARRIÈre et Marcel CourduriÉ, « Un sophisme économique... », art. cit., p. 43.

34. ACCIM LIX/1038, convention du 2 mars 1759.

35. ACCIM, LIX/204, lettre du 7 avril 1759.

36. ACCIM, LIX/525, lettre du 7 février 1759.

37. ACCIM, LIX/525, lettres du 7 février et du 7 mars 1759. 
38. « Nous avons reçû par votre dernière du 3 du court [courant] la copie de la premiere que vous avés ecrite a Mrs Garavaque et Cusson ». (ACCIM, LIX/204, lettre du 8 septembre 1759).

39. «Nous vous remettons ci joint diverses lettres ouvertes pour plusieurs amis du Languedoc ». (ACCIM, LIX/737, lettre du 10 janvier 1761).

40. Évaluation obtenue à partir des pièces comptables contenues dans ACCIM, LIX/41, comptes courants avec les correspondants dont le nom commence par la lettre $\mathrm{G}$ et dans les bilans trouvés dans LIX/1038, Maison Garavaque et Cusson, à Smyrne.

41. Cela explique les régulières occurrences des draps de Jean Jullien de cette ville au cours de la première société Garavaque et Cusson (1759-1764). Le 24 juin 1762, les régisseurs remarquaient : « Nous voyons avec plaisir les affaires que vous [les Roux] liez avec M. Jean Jullien de St-Chinian. Nous serions bien aise quelles fussent plus considérables puisque nous avons vendu jusques aujourd'huy à l'arrivée tous les draps que vous nous avez fait passer ». (ACCIM, LIX/738, lettre du 24 juin 1862).

42. ACCIM, LIX/739, lettre du 5 décembre 1763.

43. ACCIM, LIX/737, lettre du 4 mai 1761.

44. Charles CARRIÈre, Négociants... op. cit., p. 408.

45. Smyrne n'est pas une place bancaire. Aussi le bon papier se révèle-t-il souvent rare. Il s'agit d'une faiblesse de cette échelle par rapport à Constantinople, nettement plus intégrée dans le réseau de paiements multilatéraux des grands centres européens. Cf. Sevket PAMUK, A Monetary History of the Ottoman Empire, Cambridge, Cambridge University Press, 2000, p. 169.

46. ACCIM, LIX/205, lettre du $1^{\mathrm{e}}$ août 1764.

47. ACCIM, LIX/525, lettre du 7 février 1759.

48. ACCIM, LIX/739, lettre du 31 mars 1764.

49. Mark Granovetter, op. cit., p. 228.

50. ACCIM, LIX/525, lettre du 7 février 1759. Le 22 août 1759, il fait de même à propos du choix des censaux (les courtiers), laissant aux Roux le soin de «prescrire ce qu'il convient de faire la dessus ». (ACCIM, LIX/204).

51. ACCIM, LIX/738, lettres du 6 avril 1762 et du 2 janvier 1763.

52. ACCIM, LIX/737, lettre du 4 mai 1761.

53. ACCIM, LIX/738, lettre du 24 juin 1762.

54. Ibid.

55. ACCIM, LIX/739, lettre particulière du 28 avril 1764.

56. ACCIM, LIX/205, lettre du 26 mai 1764.

57. Mark GRANOVETTER, op. cit., p. 228.

58. ACCIM, LIX/205, lettre du 21 juillet 1764.

59. Cette proposition ne peut que surprendre le lecteur de la correspondance Garavaque. Les régisseurs n'ont pas de mots assez forts pour exprimer leur dégoût pour ces opérations, les affaires constantinopolitaines étant réputées pour leur extrême lenteur. Le régisseur marseillais a donc tout essayé pour impliquer davantage Paul Cusson.

60. ACCIM, LIX/739, lettre du 16 octobre 1764.

61. ACCIM, LIX/205, lettre du 22 septembre 1764.

62. ACCIM, LIX/205, lettre du 8 septembre 1764.

63. Mark GRANOVETTER, op. cit., p. 229.

64. En 1771, alors qu'il fait faillite, Paul Cusson écrit à Roux : «Nous vous avions marqué par notre précedente que la pertte de $8 \%$ de commission sur les 4000 piastres de mize de fonds à Smirne ne sçauroit nous régarder, il faut que cela soit tenu en compte a M. Gme Cusson. Les $12.000 \mathrm{ft}$ doivent nous être remises telles que nous les avons remises ». (ACCIM, LIX/206, lettre du 12 janvier 1771).

65. ACCIM, LIX/738, lettre particulière du 14 avril 1763.

66. ACCIM, LIX/738, lettre particulière du 5 décembre 1763. 
67. ACCIM, LIX/738.

68. Ibid.

69. ACCIM, LIX/203, lettre du 29 octobre 1763.

70. «J'ay apris par vous et par Cusson son retour a Marseille et le peu de succès de son voyage. J'ay toujours pensé qu'il ne serviroit qu'à le faire connoître et ce n'est pas ce qu'il y avait de plus necessaire mais ce que je trouve de déplacé dans sa façon de faire c'est d'avoir dit inconsidérément qu'il venait procurer des amis a notre maison. Je sens combien de pareils propos doivent vous avoir déplus ». (ACCIM, LIX/739, lettre particulière du 9 février 1764).

71. ACCIM, LIX/738, lettre particulière du 5 décembre 1763.

72. Ibid.

73. ACCIM, LIX/739, lettre particulière du 31 août 1765. Les trois citations suivantes sont de la même source.

74. ACCIM, LIX/1038, mémoire instructif du 2 avril 1759.

75. ACCIM, LIX/739, lettre particulière du 22 août 1764.

76. ACCIM, LIX/205, lettre du 4 août 1764.

77. « Au sujet de ces grandes dépenses, il avoue qu'il a eu tord et dore en avant il va les réduire a tel point que l'on n'aura plus des plaintes a luy faire a ce sujet. Notre sieur P. Cusson luy avoit ecrit sur un ton fort haud ». (ACCIM, LIX/205, lettre du 3 octobre 1764).

78. Sébastien LuPO, " "Vous m'avés si fort imposer de ne pas répliquer..." Réseau et hiérarchie dans une commandite marseillaise à Smyrne au XviIIe siècle», The Historical Review-La Revue Historique, $\mathrm{n}^{\circ} 7$, Institut de recherches néohelléniques (FNRS), Athènes, 2010, p. 54.

79. Ce sera le cas, et sans le moindre ménagement, en 1776 .

80. ACCIM, LIX/748, lettre particulière du 24 février 1768 .

81. ACCIM, LIX/744, lettre particulière du 12 août 1771 , la faillite de son père n'est sans doute pas étrangère à cette obséquiosité particulièrement marquée.

82. Pierre-Paul Garavaque, de retour à Marseille, a fondé "Garavaque et Latil ». Cette société associée à la maison Roux assure, selon un protocole complexe, le rôle de majeurs.

83. ACCIM, LIX/206, lettre du 16 février 1771.

84. ACCIM, LIX/730, lettre particulière de Guillaume Cusson du 9 janvier 1773.

85. «J'espere que vous n'aurés jamais a vous reprocher de me l'avoir accordée [la confiance de Roux] \& si je viens a manquer a quelque chose croyés Messieurs que les sentiments ni la volonté n'y auront jamais la moindre part. J'ay trouvé la meme façon de penser dans M. Cusson. Je ne saurais vous rendre Messieurs combien je suis content de lui a tous égards. Je ne pourrais sans injustice vous cacher qu'il rempli ses devoirs avec beaucoup d'exactitude \& de plaisir, que sa conduite est tres reguliere \& encore plus eloignée de tous sujets de depence, que si autres fois il n'a pas assés reflechi sur ce dernier objet, il ne cherche qu'a reparer les torts \& (...) en cet etat, j'ose vous prier Messieurs de vouloir bien lui accorder la signature de notre société ». (ACCIM, LIX/730, lettre particulière de Cailhol du 9 janvier 1773).

86. ACCIM, LIX/726, lettre particulière du 1er octobre 1776.

87. ACCIM, LIX/203, lettre de Cusson aîné du 14 septembre 1776.

88. ACCIM, LIX/726, lettre particulière du 1er octobre 1776.

89. Mark GRANOVETTER, op. cit., p.235.

90. Douglass NoRTH, Le processus du développement économique, Paris, Éditions d'Organisation, 2005 , p. 78.

91. «J'ai montré a Cusson lui meme et ses amis Messieurs Espanet, Marragon, Fonton etc... quel a été le sujet de sa disgrace. J'en ai offert a ces Mrs la preuve par nos livres. On l'a blamé et convenu qu'il avoit beaucoup plus de torts qu'on ne pensoit». (ACCIM, LIX/726, lettre particulière du 1er octobre 1776). 


\section{AUTEUR}

\section{SÉBASTIEN LUPO}

Doctorant en histoire moderne à l'Université d'Aix-Marseille, Laboratoire Temps, Espaces,

Langages, Europe Méridionale - Méditerranée (TELEMMe, UMR 7303), MMSH, 5, rue du Château de l'Horloge, BP 647, 13094 Aix-en-Provence cedex 2 